\title{
Editorial: Biotechnology for Tissue Engineering
}

In 2008, the issue of Open Biotechnology Journal entitled "Biotechnology for Tissue Engineering" presented nine papers focused on the biotechnology for tissue engineering.

Technologies for tissue engineering are consisted of many fields of technologies, such as cell engineering, gene technology, nano technology and biomaterials. The purpose of this issue of Open Biotechnology Journal is to highlight studies of those fields.

The first article by Echigoya et al., [1] highlights that a number of Oct4-positive human amniotic epithelial (HAE) cells can be recovered as potentially pluripotent stem cells. These cells may be useful for tissue engineering. The second article by Tamaki et al., [2] investigates candidates expressed in the apical bud, focusing on the epithelial-mesenchymal interaction during tooth development. The third article by Xu et al., [3] evaluates the effects of hydroxyapatite (HAp) on differentiation- and mineralization-related gene expression in the dental epithelial cell line. These two papers are very important for tooth tissue reconstruction. Next article by Saiga et al., [4] shows a possible in vitro model system for the analysis of relationship between our proposed p75NTR-expressing tissue-type-stem cell (TSC) candidate and HPV16-immortalized p75NTR-positive putative stem cell lines. Next article by Chen et al., [5] examines the effects of extracellular matrix (ECM) proteins and cationic polymers on the adhesion and proliferation of rat islet cells, RIN-5F cells. The results indicate that the ECM proteins of laminin, vitronectin, and type IV collagen, and cationic poly(L-lysine) will be useful for the surface modification and construction of biomaterials and scaffolds for islet cell culture and tissue engineering. Next article by Ito et al., [6] evaluates spheroid formation of pancreatic $\beta$-cell line RIN in a round bottom 96-well plate using the cell-cross linker, especially in effect of serum on spheroid formation. Next article by Kakinoki et al., [7] investigates that the surface of stainless steel (SUS316L) is modified by alternating immersion in a solution of human serum albumin (HSA) and solution of a tartaric acid derivative (TAD). The results indicate that alternating immersion in solutions of HSA and TAD is a useful technique for functionalizing the surfaces of metals. Next article by Kawazoe et al., [8] shows that chitosan/DNA polyelectrolyte complexes are useful for controlling cell spreading and aggregation. Last article by Chen et al., [9] shows new technology for DDS using gold nanoparticles.

These issues aim to stimulate future studies for biotechnology for tissue engineering.

\section{References}

[1] Echigoya T, Takeuch M, Hori H, Hirahara F, Yasumoto S. Human Amniotic Epithelium as an Unlimited Source of Oct4-Expressing Totipotent Stem Cell Subset. Open Biotechnol J 2008, 2, 102-110.

[2] Tamaki Y-T, Fujiwara N, Shibata S, Wakisaka S, Harada H. The epithelial-mesenchymal interaction plays a role in the maintenance of the stem cell niche of mouse incisors via Fgf10 and Fgf9 signaling. Open Biotechnol J 2008, 2, 111-115.

[3] Liming Xu L, Harada H, Ikoma T, Taniguchi A. Hydroxyapatite- and amelogenin protein-induced expression of mineralization-related genes in a dental epithelial cell line. Open Biotechnol J 2008, 2, 116-120.

[4] Saiga K, Takeuchi M, Kikuchi K, Morimura S, Kiguchi K, Yasumoto S. Expansion of p75NTR/Oct4-Expressing Putative Stem Cells in HPV16-Transformed Precancerous Immortal Cell Lines under the Presence of TGF $\beta$ and TNF $\alpha$. Open Biotechnol J 2008, 2, 121-132.

[5] Chen G, Kawazoe N, Tateishi T. Effects of ECM Proteins and Cationic Polymers on the Adhesion and Proliferation of Rat Islet Cells. Open Biotechnol J 2008, 2, 133-137.

[6] Ito M, Taguchi T. The effect of serum on the PEG-based crosslinker-induced spheroid formation of pancreatic $\beta$-cell. Open Biotechnol J 2008, 2, 138-142.

[7] Kakinoki S, Katada Y, Uchida Y, Taguchi T. Surface Modification of SUS 316L Stainless Steel with Tartaric Acid Derivative-Crosslinked Human Serum Albumin Matrices. Open Biotechnol J 2008, 2, 143-147.

[8] Kawazoe N, Narita Y, Chen G, Satomi T, Tateish T. Chitosan/DNA Polyelectrolyte Complex Membranes for Controlling Cell Spreading and Aggregation. Open Biotechnol J 2008, 2, 148-151.

[9] Chen G, Takezawa M, Kawazoe N, Tateishi T. Preparation of Cationic Gold Nanoparticles for Gene Delivery. Open Biotechnol J 2008, 2, 152-156. 